\title{
Short Communication: Flexure delicacies
}

\author{
S. Henein \\ Centre Suisse d'Electronique et de Microtechnique (CSEM SA), Neuchâtel, Switzerland \\ Correspondence to: S. Henein (simon.henein@csem.ch)
}

Received: 27 July 2011 - Revised: 22 December 2011 - Accepted: 9 January 2012 - Published: 13 January 2012

\begin{abstract}
Flexures are nowadays enjoying a new boom in numerous high-precision and extreme-environment applications. The paper presents some delicate issues concerning stiffness compensation, large reduction ratios, as well as rectilinear and circular movements in compliant mechanisms. Novel concrete technical solutions to these well-known issues are described, giving a glimpse into the vast and still largely unexploited potential of flexure mechanisms manufactured by wire-electrical-discharge machining.
\end{abstract}

\section{Introduction}

Although the basic principles of flexible bearings have been known for several decades (Koster, 1996), the design methods that could be found in literature have long remained fragmented. Only recently the interest for flexures and their applications has grown, leading to a more systematic approach of the respective design methodologies such as Smith (2000), Henein (2001) and Howell (2001). This development has been driven by the increasing need for motion accuracies in the nanometer range in extreme environments like vacuum, cryogenic or high temperatures, under radiation or in outer space. Moreover, the increasing prevalence of manufacturing technologies like wire-electrical-discharge machining (wireEDM) and more recently of deep silicon etching has made flexure-mechanisms a key element of the broad technological system our society relies upon for living.

Typical limitations of flexure mechanisms are the presence of an elastic restoring force that opposes the intended motion, the large outer dimensions of the mechanisms relative to the admissible strokes, and the complex kinematic movements of the most basic flexures (e.g. parallel spring stage and cross spring pivot) which do not produce purely rectilinear or circular trajectories. The three sections below illustrate some simple, yet subtle, solutions addressing these three issues: first a zero-stiffness translation bearing is presented, then a virtual lever with a very large reduction ratio (up to 1:1000) and finally a rectilinear translation mechanism and a circular flexure pivot.

\section{Stiffness compensation and bi-stability}

Ideal bearings would be movable by infinitesimal forces or torques. However, this is not the case for basic flexures, which present a non-zero spring constant that resist motion intended away from their neutral position. Nevertheless, if a preloaded spring is used in an arrangement where the spring loses elastic energy while the flexure is moved away from its neutral position, then the overall stiffness of the structure can be reduced (Koster, 1996; Henein, 2001). Special designs allow to practically reduce the natural stiffness of flexures by several orders of magnitude (Fig. 1), approaching a zero-stiffness ideal behaviour. If additional preload is applied, then negative stiffness can be achieved, i.e. the flexures present a bi-stable behaviour. Figure 1 shows an example of such a mechanism composed of a fixed frame (a), and a main parallel spring stage (b) defining the trajectory of the mobile block (c). A secondary parallel spring stage (d) is attached to (c), and both stages are loaded in compression by a preload spring (e). A fifth blade (or rod) (f) is used to define the position of the secondary stage. One can give the following qualitative explanation to the behaviour of this mechanism: when (c) is moved away from its neutral position, the four blades (b and d) store elastic energy. Meanwhile the elongation of the preload spring decreases, due to the shortening effect of both parallel spring stages, therefore the preload spring loses elastic energy. At the level of the mechanism, it is the global balance between the rates of elastic energy variations of the parallel spring blades versus the preload spring which dictates the elastic behaviour of the mechanism.

For simplification, the elastic element (f) might be combined with (e) as a single preloaded blade. This leads to a planar mechanism that can be manufactured monolithically 

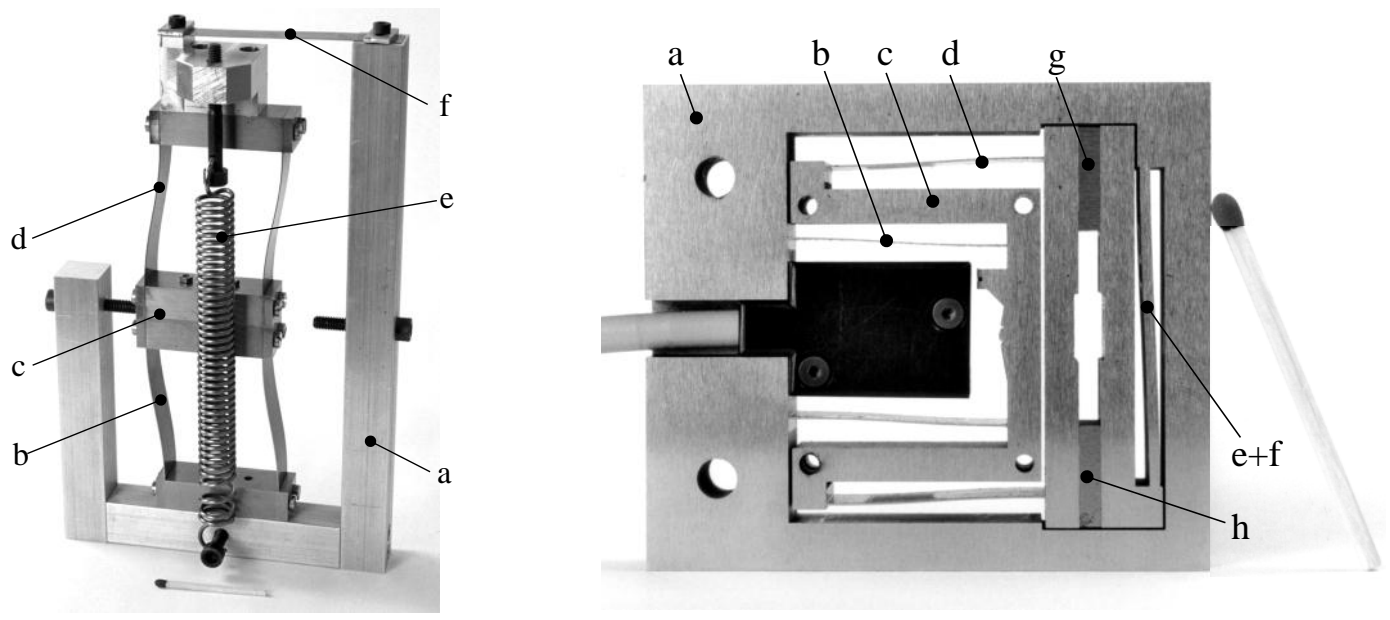

Figure 1. Parallel spring stage equipped with a stiffness compensation mechanism. Left: mock-up model showing the architecture. Right: same arrangement machined monolithically by wire-electrodischarge machining (wire-EDM).

(Fig. 1). In this arrangement, the middle-point of the blade $(e+f)$ is placed on the axis of symmetry of the mechanism. Therefore, the insertion of the preloading shims $(\mathrm{g})$ and $(\mathrm{h})$ result in an equal compression loading of all four guiding blades (b and d). By choosing the right shim thickness, the preload might be tuned to obtain a reduced stiffness, a near zero-stiffness or a negative stiffness. Equations (1) and (2) give the formulas for the calculation of the theoretical preload force which leads to zero-stiffness (Henein, 2001).

In a parallel spring stage, the linear stiffness $k$ of one blade of length $L$, width $b$, thickness $h$ and Young's Modulus $E$, preloaded in compression by a force $N$ is:

$k=\frac{N}{\frac{2}{S} \tan \frac{S L}{2}-L} ;$ with $S=\sqrt{\frac{N}{E I}} ;$ and $I=\frac{b h^{3}}{12}$

Therefore a preload $N_{0}$ exists, which leads to a zero stiffness of the blade:

$N_{0}=\frac{\pi^{2} E I}{L^{2}}$

Figure 2 shows the theoretical force versus displacement characteristic of the monolithic mechanism shown in Fig. 1 with and without preloading the blade $(e+f)$. The dimensions $(L, b, h)$ of the four guiding blades (b and $\mathrm{d})$ are $26 \times 10 \times 0.25 \mathrm{~mm}$. The dimensions of the preload blade $(\mathrm{e}+\mathrm{f})$ are $32 \times 10 \times 1.1 \mathrm{~mm}$. The material used is a spring steel with a Young's modulus of $196 \mathrm{GPa}$. The nominal preload force in the neutral position is $5 \%$ above the load $N_{0}$ (Eq. 2), which leads to a slightly negative stiffness around the central position (i.e. with compensation, the slope of the curve is negative around the origin). The stiffness reduction effect is clearly visible on the graph.

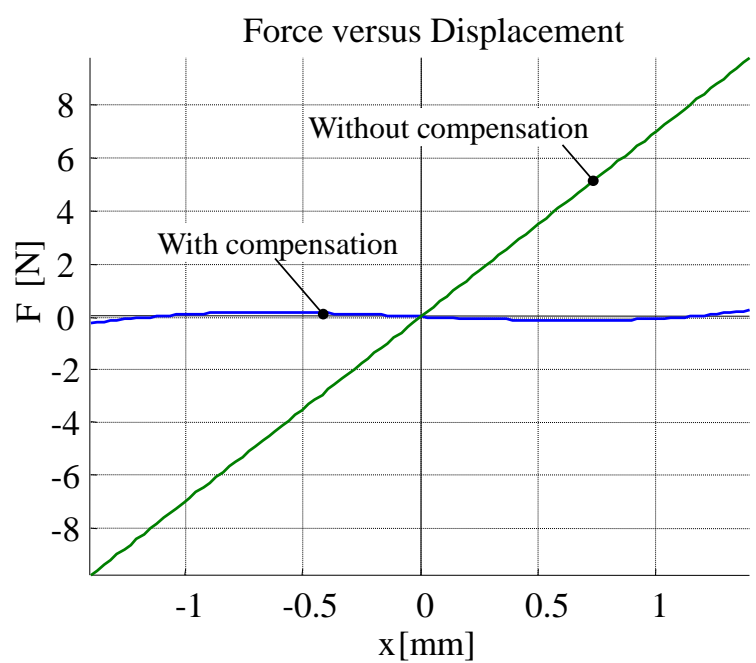

Figure 2. Force applied on the mobile block (c) (Fig. 1, right) as a function of its displacement with and without stiffness compensation.

\section{Achieving large reduction ratios}

Striving after nanometric motion accuracies often leads to the use of mechanical transmissions with very large reduction ratios, which scale down the motion of commercial actuators. It is well-known in the state-of-the-art that flexures can be used as reduction mechanisms, but the classical solutions generally have non-linear characteristics (i.e. the reduction factor is not constant over the motion range) (Eden, 1956). In comparison, a non-conventional design such as the Nanoconverter (Fig. 3) (Henein, 2006) presents the following key advantages: it exhibits a constant reduction factor; it can achieve very high reduction factors (typically up to $1: 1000$ ); it can be designed to be tunable using a simple tuning screw 

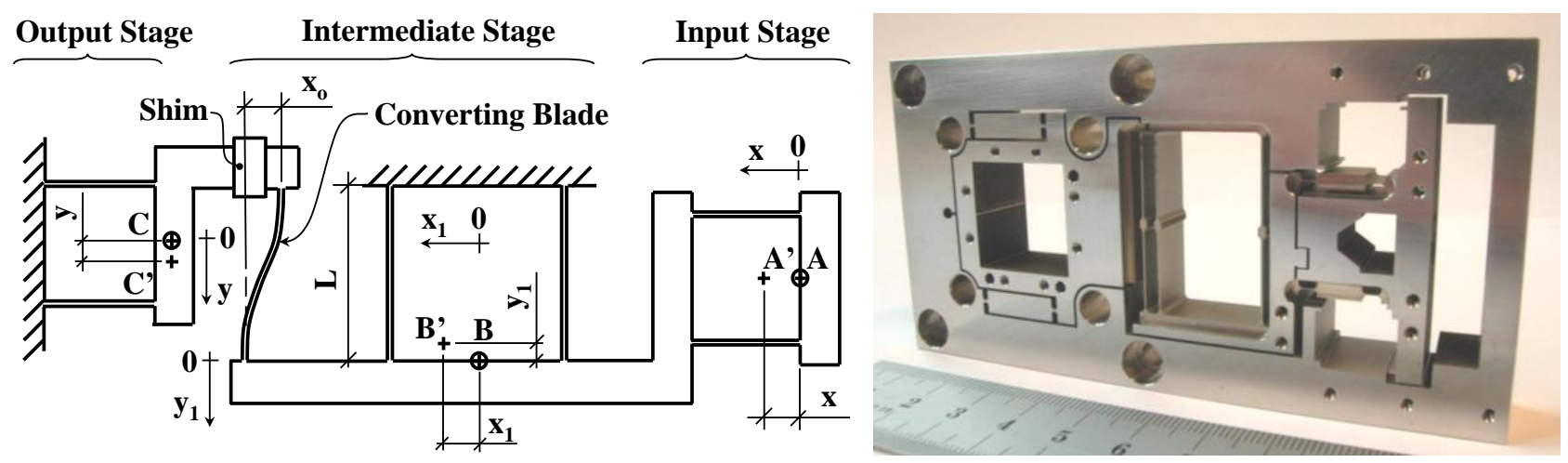

Figure 3. Working principle (using an offset shim) and photo of a monolithic Nanoconverter.
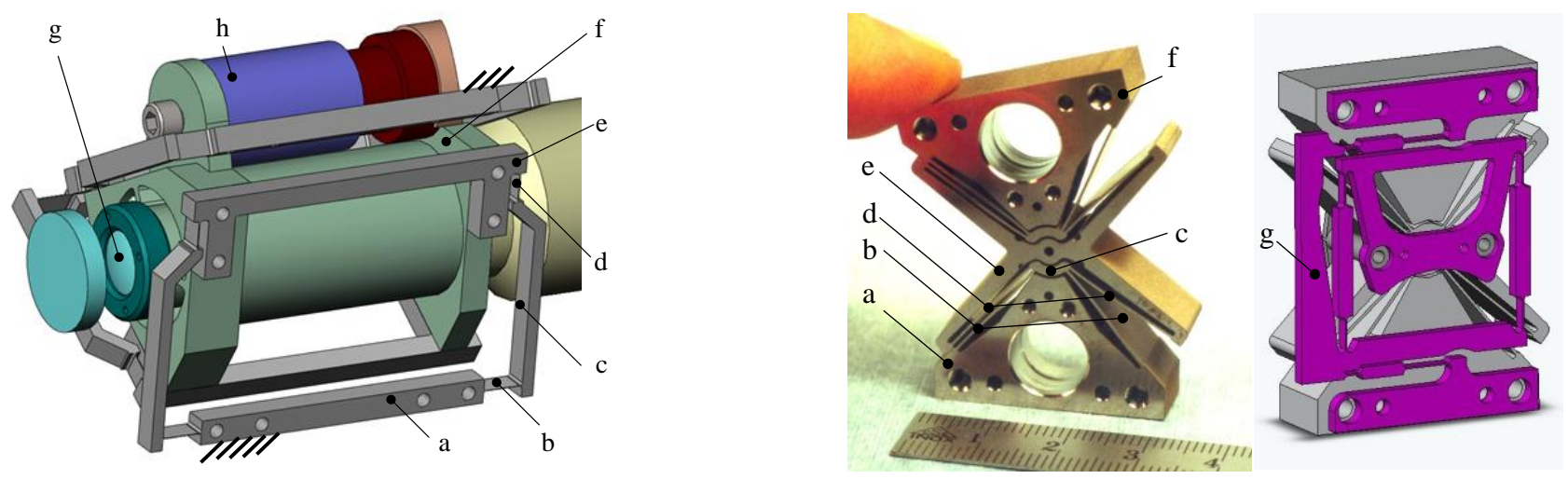

Figure 4. Left: rectilinear flexure mechanisms of the Mars Close-up Imager. Center: picture of the monolithic Butterfly flexure pivot (angular stroke up to $\pm 15^{\circ}$; centre shift below 2 microns). Right: Butterfly Pivot equipped with a slaving plate.

or shim to select the reduction factor over a wide range (typically 1:20 to 1:1000); its simple planar structure can be manufactured monolithically (no need for assembly) using a wide variety of techniques (e.g. wire-EDM, laser cutting, silicon etching, LIGA).

The working principle is the following: a commercial linear actuator with micrometric motion accuracy rectilinearly drives point A (Fig. 3) of the Input Stage to A'. This motion is transmitted to the Intermediate Stage that is guided by a classical parallel-spring-stage (with blade length $L$ ): point B moves to B'. Due to the shortening of the blade projection, the motion of this stage is a well-known parabolic translation: $y_{1}=-3 x_{1}^{2} /(5 L)$, where $x_{1} \cong x$. A third blade of the same length $L$ (called "Converting Blade"), that has an offset deformation $x_{0}$, links the Intermediate Stage to the Output Stage. The Output Stage is guided vertically by a classical parallel-spring-stage. The motion $x_{1}$ causes the Converting Blade to shorten, following the same parabolic law as the two blades of the Intermediate Stage, but with an offset $x_{0}$. The resulting motion $y$ (Eq. 3) of the Output Stage (motion from $\mathrm{C}$ to $\mathrm{C}^{\prime}$ ) is equal to the differential shortening of the blades (subtraction of two parabolas with an offset):

$$
\begin{aligned}
& y=\frac{3\left(x+x_{0}\right)^{2}}{5 L}-\frac{3 x^{2}}{5 L}=\frac{6 x_{0}}{5 L} x+\frac{3 x_{0}^{2}}{5 L} ; \\
& i=\frac{x}{y}=\frac{5 L}{6 x_{0}}
\end{aligned}
$$

Therefore, if the origin of the $\mathrm{y}$-axis is adequately chosen, the displacement $y$ of the Output Stage is simply proportional to the displacement $x$ of the actuator, with reduction ratio $i$ (Eq. 4) that is constant over the whole displacement range and is inversely proportional to the offset $x_{0}$. Choosing an offset $x_{0}$ that is small compared to the blade length $L$ leads to very large demagnification ratios. This is mechanically very easy to achieve by using a shim or by monolithic manufacturing as in Fig. 3.

\section{Rectilinear and circular flexures}

Producing purely rectilinear movements can be achieved with the well-known compound-parallel-spring-stage (Smith, 2000), but some other less common structures exist like the 5 or 6 folded leaf springs (Schellenkens et al., 1998). For example, the latter concept has been adapted for the 
Close-up Imager (CLUPI Instrument) (Fig. 4) of the European Mars Mission EXOMARS developed by CSEM for the Space Exploration Institute (SPACE-X). It is composed of three identical plates connecting the fixed outer frame to the mobile body (f) holding the optical lens (g). Each plate is a planar monolithic symmetric compliant structure composed of a fixed bar (a), a first flexure hinge (b), a second rigid bar (c), a second flexure hinge (d) and an output bar (e). Each plate blocks one rotation and one translation (f). The three thin plates can accommodate for the over-constraint of this kinematic arrangement without causing high stresses. Hence, this arrangement offers long stroke rectilinear motion.

Nearly pure circular rotations can be achieved by structures such as the Butterfly Pivot (Henein et al., 2003; Pei and $\mathrm{Yu}, 2011)$. This monolithic planar compliant mechanism (Fig. 4) was originally designed for space pointing applications. It is composed of a fixed base block (a), two blades (b) forming a first Remote-Center-Compliance (RCC) pivot, an intermediate block (c), a second RCC pivot (d) that compensates for a large part of the parasitic shifts produced by (b), a second intermediate block (e), then a mirror arrangement leading to the output block (f) doubles the total angular stroke. An optional internal coupling mechanism (g) can be integrated in order to suppress the undesired internal DOF of the central cross (e) raising its Eigen-mode from 320 to $2000 \mathrm{~Hz}$. This additional kinematic chain plays the same role as the slaving lever commonly used for the same reason with the compound parallel spring stage (Smith, 2000), i.e. it forces the intermediate body (e) to move by exactly half the stroke of the output (f).

\section{Conclusions}

During the last decades, the old and simple idea of using the elastic deformation of solid bodies to produce well defined movements has been used in more and more application fields. As the simplest original components (e.g. cross spring-pivots and leaf spring stages) are still widely used, many novel designs are flourishing which exploit the possibilities offered by wire-EDM to produce mechanical structures with original characteristics like zero-stiffness, extreme reduction ratios or ideal kinematic behaviours. This illustrates how this new manufacturing technology has led not only to an improvement of existing concepts but also to a revolution in the design of flexure-mechanisms. In this light, one should be very attentive to the coming revolution in this field, which will probably come from the use of deep silicon etching technologies in the design of tomorrow's flexuremechanisms.
Acknowledgements. The author wishes to thank J.-L. Josset from the Space Exploration Institute, Ph. Schwab, L. Giriens, W. Glettig and P. Spanoudakis from CSEM, U. Frommherz from the Paul Scherrer Institut, as well as S. Bottinelli from MECARTEX SA for their contributions to the work presented in this short communication.

Edited by: J. A. Gallego Sánchez

Reviewed by: M. Richard and another anonymous referee

\section{References}

Eden, E. M.: Application of spring strips to instrument design, Notes of Applied Science No. 15, 1956.

Henein, S.: Conception des guidages flexibles, Presses Polytechniques et Universitaires Romandes, ISBN 2-88074-481-4, 2001.

Henein, S.: Device for converting a first motion into a second motion responsive to said first motion under a demagnification scale, Patent EP06021785, Holder: Paul Scherrer Institut, 2006.

Henein, S., Spanoudakis, P., Droz, S., Myklebust, L., and Onillon, E.: Flexure pivot for aerospace mechanisms, Proc. 10th European Space Mechanisms and Tribology Symposium, San Sebastian, Spain, 24-26 September 2003, ESA SP-524, 285-288, 2003.

Howell, L.: Compliant mechanisms, John Wiley \& Sons Inc, 2001.

Koster, M. P.: Constructieprincipes voor het nauwkeurig bewegen en positioneren, 5th edn., HBuitgevers, Baarn, NL, 1996.

Pei, X. and Yu, J.: ADLIF: a new large-displacement beam-based flexure joint, Mech. Sci., 2, 183-188, doi:10.5194/ms-2-1832011, 2011.

Schellenkens, P., Rosielle, N., Vermeulen, M., Wetzels, S., and Pril, W.: Design for precision: current status and trends, Annals of the CIRP, Vol. 47/2/1998, 1998.

Smith, S. T.: Flexures, Gordon and Breach Science Publishers, ISBN 90-5699-261-9, 2000. 